\title{
Identification of TPS and TPP gene families in Cannabis sativa and their expression under abiotic stresses
}

\author{
J. SUN ${ }^{1,2,+}$ (D) Z.G. DAI ${ }^{2,+}$, X.Y. ZHANG ${ }^{2}$, Q. TANG ${ }^{2}$, C.H. CHENG ${ }^{2}$, C. LIU², Y. YU ${ }^{2}$, G.C. XU², \\ D.W. XIE ${ }^{1, *}(\mathbb{D})$, and J.G. SU ${ }^{2, *}$ (ID \\ ${ }^{1}$ School of Life Sciences, Nantong University, Nantong 226019, P.R. China \\ ${ }^{2}$ Institute of Bast Fiber Crops, Chinese Academy of Agricultural Sciences, Changsha 410205, P.R. China
}

*Corresponding authors: E-mails: xiedongwei@163.com,su_changsha@163.com

\begin{abstract}
Trehalose is a nonreducing disaccharide that is involved in the regulation of plant responses to a variety of environmental stresses. Trehalose 6-phosphate synthase (TPS) and trehalose 6-phosphate phosphatase (TPP) are two key enzymes in trehalose synthesis and they are widely distributed in higher plants. At present, TPS family genes have been systematically identified and analyzed in many plant species, but the TPP family genes have been rarely studied. In this study, ten TPS and six TPP genes in cannabis (Cannabis sativa L.) were identified at the genomic level. The phylogenetic tree of TPS and TPP family members in cannabis, Arabidopsis, and rice was constructed, and all the genes were divided into three subgroups: Class I, Class II, and Class III. The number of exons and motif types among Class I members was exactly the same, as were Class II members, but the gene structure and motif types of Class III members were slightly different. There were four pairs of CSTPSs and CSTPPS that had gene duplication, indicating that gene duplication events played an important role in the amplification of TPS and TPP families in cannabis. The results of expression analysis under abiotic stresses showed that $68.75 \%$ of $C S T P S$ and $C S T P P$ genes were significantly induced by at least one abiotic stress. Among these genes, the expression of CsTPS1, CsTPS9, and CsTPPA was highest under at least one abiotic stress. These three genes may play a key role in abiotic stress responses. Most of the CsTPS and CsTPP genes that are closely located in the evolutionary tree have the same or similar functions. To our knowledge, this is the first paper that systematically reports the TPS and TPP gene families in cannabis.
\end{abstract}

Keywords: Cannabis sativa, cold, drought, phylogenetic tree, salt stress, TPP and TPS gene families, trehalose.

\section{Introduction}

Trehalose is a nonreducing disaccharide that is widely distributed in organisms and has different biological functions in different species. In plants, trehalose is involved in the regulation of the response to a variety of environmental stresses (Paul et al. 2008). Trehalose has a stronger ability to bind water than other sugars (Lerbret et al. 2005). Trehalose can maintain the biological structure and function of biomolecules by replacing water, concentrating water around biomolecules or in the form of a vitrification agent under the conditions of water shortage or freezing (Sundaramurthi et al. 2010, Hackel et al. 2012). Because trehalose has a strong anti dehydration effect,

$\overline{\text { Received }} 16$ April 2021, last revision 6 August 2021, accepted 25 August 2021.

Abbreviations: CBD - cannabidiol; G6P - glucose-6-phosphate; Ka - nonsynonymous substitution rate, Ks - synonymous substitution rate; $\mathrm{Mr}$ - relative molecular mass; $\mathrm{pI}$ - isoelectric point; T6P - trehalose 6-phosphate; TPP - trehalose 6-phosphate phosphatase; TPS trehalose 6-phosphate synthase; UDPG - UDP glucose.

Acknowledgements: This work was supported by the Basic Science Research Project of Nantong City (JC2020156), the National Natural Science Foundation (31801409), the Special Construction of Modern Agricultural Industrial Technology System (CARS-16-E01), the Protection and Utilization of Crop Germplasm Resources (2016NWB044), and the National Science and Technology Resource Sharing Service Platform Project (NCGRC-2020-15).

${ }^{+}$These authors contributed equally.

Conflict of interest: The authors declare that they have no conflict of interest. 
it can protect biofilms and proteins from damage under drought, cold, high salinity, and other stress conditions.

The pathway of trehalose synthesis in higher plants is relatively clear and includes two main steps of enzymatic reactions. First, trehalose 6-phosphate synthase (TPS) catalyzes UDP glucose (UDPG) and glucose-6-phosphate (G6P) to produce trehalose 6-phosphate (T6P), and then trehalose 6-phosphate phosphatase (TPP) catalyzes the dephosphorylation of T6P to produce trehalose (Avonce et al. 2006). In the above synthetic pathway, two key enzymes, the TPS enzyme encoded by TPS genes, catalyze the biosynthesis of T6P, and the TPP enzyme encoded by TPP genes catalyzes the biosynthesis of trehalose. TPS and TPP enzymes are widely distributed in higher plants. To date, systematic identification and analysis of TPS family genes have been performed in rice (Ge et al. 2008), Arabidopsis (Yang et al. 2012), Populus (Yang et al. 2012), wheat (Xie et al. 2015), lotus (Jin et al. 2016), cotton (Mu et al. 2016), cassava (Han et al. 2016), potato (Xu et al. 2017), apple (Du et al. 2017), drumstick tree (Lin et al. 2018), Brachypodium distachyon (Wang et al. 2019), sugarcane (Hu et al. 2020), Prunus mume (Yang et al. 2020), and grapevine (Morabito et al. 2021). Some genes show potential functions under stress conditions, but their expression patterns are also diverse. In the field of the TPP family, genes have been identified in rice (Ge et al. 2008), Arabidopsis (Vandesteene et al. 2012), cassava (Han et al. 2016), and Brachypodium distachyon (Wang et al. 2019). The TPP gene family in plants has been studied less than the TPS gene family.

Cannabis (Cannabis sativa L.) has been an economically important crop since ancient times (Skoglund et al. 2013). Its rich phytochemicals can be used in the field of medicine, and its high-quality fibre is widely used in textiles, building materials, chemicals, and energy. In addition, cannabis also has good drought resistance and insect resistance. Its developed roots can fix the soil and prevent soil erosion, and compared with other crops, its water demand is also rather low (Andre et al. 2016). The above shows that cannabis has good stress resistance and adaptability, and is easy to be cultivated so that its various functions can be widely used. In recent years, cannabidiol (CBD) has been proven to play an important role in the treatment of schizophrenia, epilepsy, neurodegenerative diseases, multiple sclerosis, emotional disorders and other nervous system diseases, which has caused worldwide attention, with the demand for cannabis increasing dramatically (Pretzsch et al. 2019).

Abiotic stresses such as drought, low temperature, and soil salinity are the main factors affecting crop growth and yield reduction (Vij et al. 2010). Drought and high concentrations of $\mathrm{NaCl}$ can reduce the soil water potential, thus reducing the water absorption by the roots. Salt stress inhibits crop growth by causing ion imbalance and osmotic stress (Dong et al. 2020). Low temperature and chilling injury can delay the growth period of crops, resulting in yield decline (Zhang et al. 2014). Abiotic stresses are major threats to global agriculture and an important reason leading to the reduction of cannabis production (Mahajan et al. 2005, Hu et al. 2019). In this study, evolution, gene structure, and gene duplication analyses of the TPS and TPP gene families in cannabis were conducted, and the expression of selected genes under abiotic stress was analyzed by RT-qPCR. The purpose of this study was to identify the TPS and TPP gene families of cannabis and understand their functions under abiotic stresses to lay a foundation for future understanding of stress resistance mechanisms in cannabis.

\section{Materials and methods}

Identification and basic information for cannabis TPS and TPP gene family members: The TPS and TPP protein sequences of Arabidopsis thaliana were used as query sequences to search and screen the genome of cannabis (https://www.ncbi.nlm.nih.gov/assembly/ GCF 900626175.2/) by BLASTP applying default parameters. The candidate protein sequences were submitted to Pfam (http://pfam.xfam.org/) for verification, and the sequences exhibiting incomplete domains were deleted. Finally, all genes of the TPS and TPP families in cannabis were obtained. The nucleic acid sequence, coding sequence (CDS) and protein sequences were obtained from the cannabis genome. The relative molecular mass $(\mathrm{Mr})$ and isoelectric point $(\mathrm{pI})$ of the proteins were predicted in ExPasy (https://web.expasy.org/protparam). The subcellular localization of each member of the CsTPS and $C S T P P$ families was predicted by the CELLO server (http://cello.life.nctu.edu.tw).

Phylogenetic analysis: All TPS and TPP genes of Arabidopsis and rice were obtained from the Arabidopsis genome database (https://www.arabidopsis.org/) and rice genome database (http://rice.plantbiology.msu.edu/). The TPS and TPP proteins of cannabis, Arabidopsis, and rice were aligned by Clustal $W$ software. The phylogenetic tree was constructed by the neighbor-joining (NJ) method of MEGA 7.0 software, and bootstrap analysis was carried out. The repeat value was set to 1000 , and other parameters were set to default values.

Gene structure and conserved motifs: In GSDS 2.0 online software (http://gsds.cbi.pku.edu.cn/), the gene and CDSs were input to analyze and map the gene structure of CsTPS and CSTPP family members. The online software MEME (https://meme-suite.org/meme/tools/meme) was used to predict and analyze the motifs of the CsTPS and CsTPP genes, and the number of motifs was set to 20 .

Gene duplication and substitution rate analysis: The plant genome duplication database (https://popgenie.org/ node/42) was used to analyze the duplication of each gene, and the maximum distance between the duplicated genes was $500 \mathrm{~kb}$. The length of each chromosome and the position of CsTPS and CsTPP genes on the chromosome were obtained from the cannabis genome. TBtools software (Chen et al. 2020) was used to construct the collinearity analysis map of CsTPS and CsTPP genes and chromosomes. 
According to the CDS of the duplicated gene pairs, the nonsynonymous substitution rate $(\mathrm{Ka})$, synonymous substitution rate $(\mathrm{Ks})$, and $\mathrm{Ka} / \mathrm{Ks}$ were calculated by DnaSP 5.0 software (Librado and Rozas 2009). The formula $\mathrm{T}=\mathrm{Ks} / 2 \mathrm{r}$ was used to estimate the divergence time of duplicated gene pairs. The $\mathrm{r}$ value was $1.5 \times 10^{-8}$ for dicots (Yang et al. 2020).

Plants and stress treatments: The seeds of cannabis (Cannabis sativa L.) cv. DMG245 were sown in a seedling tray. The seedling tray was $26 \mathrm{~cm}$ in length, $26 \mathrm{~cm}$ in width, and $10 \mathrm{~cm}$ in height with 25 holes. Three seeds were sown in each hole, and two seedlings were pulled out after emergence to ensure one seedling in each hole. The seedlings were cultured in an artificial climate room - the day/night temperatures were $24{ }^{\circ} \mathrm{C} / 16^{\circ} \mathrm{C}$, a 16-h photoperiod, an irradiance of $300 \mu \mathrm{mol} \mathrm{m} \mathrm{m}^{-2} \mathrm{~s}^{-1}$, and relative humidity of $65 \%$. Abiotic stress treatments were carried out when the seedling heights were approximately $20 \pm 0.5 \mathrm{~cm}$. A total of four seedling trays, each containing 25 seedlings, were used for salt, drought, low temperature, and control treatment. $\mathrm{NaCl}$ solution at a concentration of $3.0 \%$ was placed in the seedling tray for salt stress treatment, and polyethylene glycol (PEG)6000 at a $20 \%$ concentration was used to mimic drought stress. The seedlings were transferred to an incubator for a $4{ }^{\circ} \mathrm{C}$ low-temperature treatment, and the seedlings under normal growth conditions were used as controls. One leaf was cut at $0,8,16$, and $24 \mathrm{~h}$ after the beginning of treatment, respectively. Three seedlings were taken from each treatment as three repetitions. The cut seedlings were quickly put into liquid nitrogen and then stored at $-80^{\circ} \mathrm{C}$ for total RNA extraction.

Quantitative real-time PCR: TRIzol (Invitrogen, Carlsbad, CA, USA) was used to extract the total RNA from cannabis, and a NanoDrop 2000 (Thermo Scientific, Wilmington, USA) was used to detect the quality, purity, and integrity of RNA. The RNA of each sample was reverse transcribed into cDNA by using the Prime $\mathrm{HiFi}$ $M M L V$ cDNA kit (CWBIO, Beijing, China).

RT-qPCR primers for the CsTPS and CsTPP genes were designed by Primer Premier 5.0 software (Table 1 Suppl.). The RT-qPCR system $\left(20 \mathrm{~mm}^{3}\right)$ consisted of $0.5 \mathrm{~mm}^{3}$ UltraSYBR One Step EnzymeMix (CWBIO), $10 \mathrm{~mm}^{3}$ of buffer, $0.5 \mathrm{~mm}^{3}$ of upstream and downstream specific primers, $1 \mathrm{~mm}^{3}$ of cDNA template, and $7.5 \mathrm{~mm}^{3}$ of $\mathrm{ddH}_{2} \mathrm{O}$. The reaction procedure was $95^{\circ} \mathrm{C}$ for $5 \mathrm{~min}, 45$ cycles of $94{ }^{\circ} \mathrm{C}$ for $10 \mathrm{~s}, 60^{\circ} \mathrm{C}$ for $20 \mathrm{~s}$, and $72{ }^{\circ} \mathrm{C}$ for $20 \mathrm{~s}$. ACTIN was used as an internal reference gene (Hu et al. 2019). The relative expression of each gene was calculated using the $2^{-\Delta \Delta \mathrm{Ct}}$ method (Livak and Schmittgen 2001).

Statistical analyses: In the RT-qPCR experiment, mean values and standard deviations (SD) were obtained from three replicates. Statistical significance was performed using a Student's $t$-test at $P \leq 0.05$ and $P \leq 0.01$ (SPSS 21.0, Chicago, IL, USA) to evaluate the difference of relative gene expressions among at $0 \mathrm{~h}$ and other time points.

\section{Results}

The Arabidopsis TPS and TPP protein sequences were used as query sequences to blast the genome of cannabis in NCBI. The aligned cannabis TPS and TPP genes were submitted to the Pfam database. After eliminating the redundant sequences without typical TPS and TPP domains, ten cannabis TPS and six TPP genes were obtained. The ten CsTPSs contained the Glyco_transf_20 (PF00982) domain and contained the trehalose PPase (PF02358) domain. The six CsTPPs only contained the Trehalose_PPase

Table 1. Basic information about CsTPSs and CsTPPS in cannabis. Chr - chromosome, aa - amino acids, Mr - molecular mass, pI isoelectric point, SL - subcellular localization.

\begin{tabular}{llrlllll}
\hline Gene name & NCBI accession & Chr & \multicolumn{1}{l}{ Location } & Number of aa & $\mathrm{Mr}[\mathrm{kDa}]$ & $\mathrm{pI}$ & SL \\
\hline CsTPS1 & XP_030490413 & 1 & $88260818 . .88281140$ & 928 & 104.61 & 6.37 & cytoplasm \\
CsTPS2 & XP_030480449 & 7 & $68900724 . .68907646$ & 949 & 106.91 & 6.15 & cytoplasm \\
CsTPS3 & XP_030508575 & 9 & $177011 . .184079$ & 817 & 93.32 & 6.97 & cytoplasm \\
CsTPS4 & XP_030484585 & 8 & $49637121 . .49641495$ & 836 & 95.38 & 5.63 & nucleus \\
CsTPS5 & XP_030500602 & 5 & $83521789 . .83526035$ & 861 & 97.34 & 5.40 & cytoplasm \\
CSTPS6 & XP_030506745 & 9 & $57346939 . .57350893$ & 856 & 96.57 & 5.86 & cytoplasm \\
CsTPS7 & XP_030496147 & 10 & $52857530 . .52864078$ & 854 & 96.69 & 5.93 & plasma membrane \\
CsTPS8 & XP_030493634 & 3 & $13012251 . .13017444$ & 858 & 96.89 & 6.22 & cytoplasm \\
CsTPS9 & XP_030510183 & 6 & $4517436 . .4521020$ & 868 & 97.55 & 5.87 & cytoplasm \\
CSTPS10 & XP_030499885 & 4 & $3333932 . .3338557$ & 862 & 96.49 & 5.75 & cytoplasm \\
CSTPPA & XP_030489124 & 1 & $3557033 . .3562147$ & 385 & 43.25 & 8.35 & chloroplast \\
CsTPPB & XP_030501902 & 5 & $74809140 . .74814109$ & 392 & 44.85 & 7.77 & mitochondrion \\
CsTPPC & XP_030500530 & 5 & $4669812 . .4678812$ & 347 & 38.97 & 7.70 & nucleus \\
CSTPPD & XP_030481171 & 10 & $94256116 . .94258867$ & 379 & 42.46 & 9.11 & nucleus \\
CSTPPE & XP_030499315 & 4 & $49532570 . .49535876$ & 374 & 41.96 & 9.26 & nucleus \\
CSTPPF & XP_030488733 & 1 & $69650913 . .69655396$ & 330 & 37.33 & 7.61 & cytoplasm \\
\hline
\end{tabular}




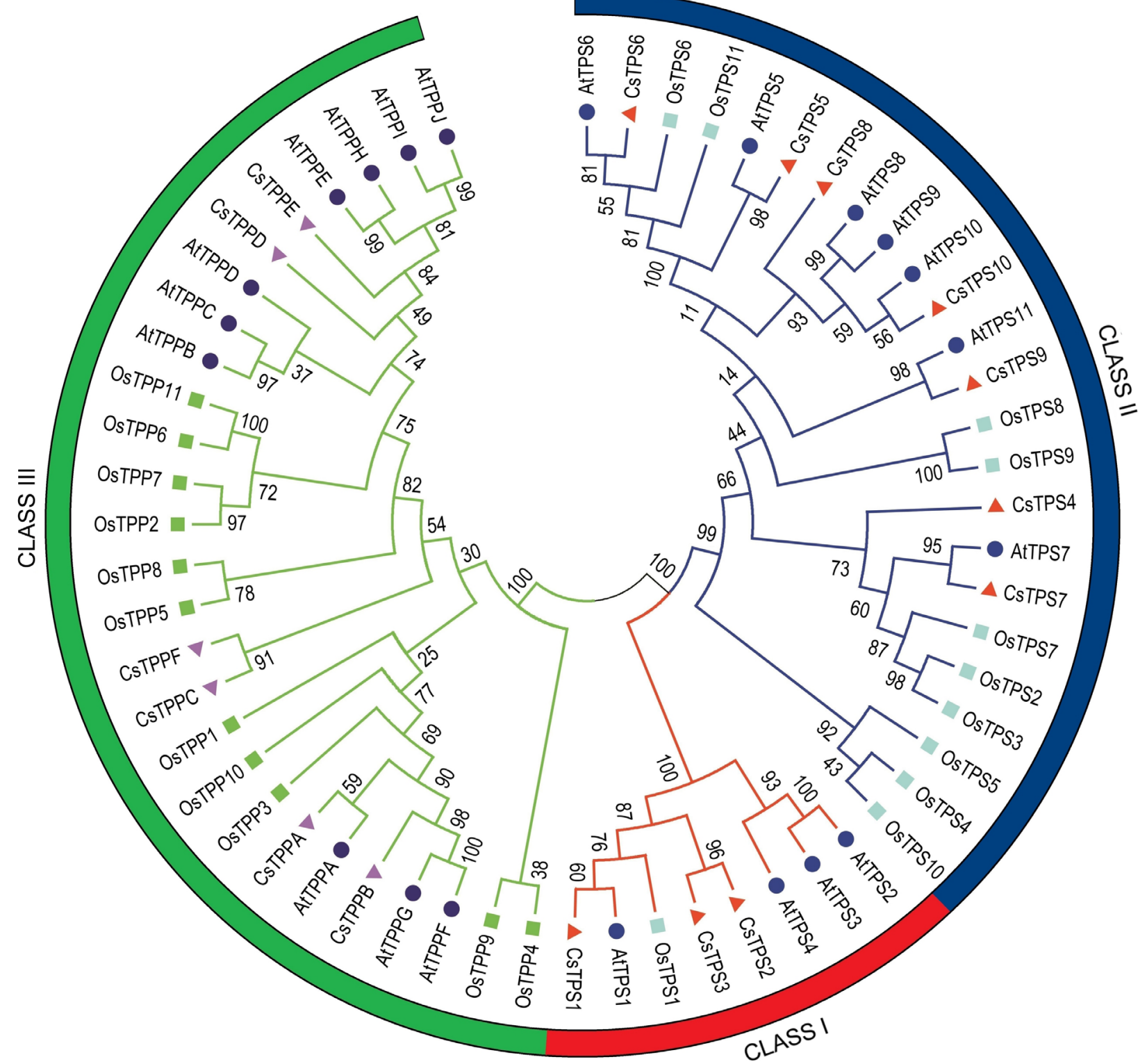

Fig. 1. Phylogenetic tree of TPS and TPP family genes in cannabis, Arabidopsis, and rice. MEGA 7.0 (bootstrap value $=1000$ ) was used to create a maximum likelihood tree and display the bootstrap value of each branch. Red, blue, and green represent Class I, Class II, and Class III, respectively. Triangle represent cannabis, circles represent Arabidopsis, and squares represent rice.

domain. The TPS and TPP genes of cannabis were named CSTPS1-10 and CSTPPA-F (Table 1), respectively, according to the homologous relationship with the TPS and TPP genes of Arabidopsis in the phylogenetic tree (Fig. 1).

ExPASy was used to predict the basic physical and chemical properties of TPS and TPP family members in cannabis (Table 1). The amino acid length of CsTPSs was 817 - 949 aa and that of CsTPPs was 330 - 392 aa. The $\mathrm{Mr}$ of the CsTPS protein was 93.32 - $106.91 \mathrm{kDa}$ and that of the CsTPP protein was $37.33-44.85 \mathrm{kDa}$. CsTPS protein was significantly larger than CsTPP protein. The pIs of CsTPS and CsTPP were 5.40 - 6.97 and $7.61-9.26$, respectively. The results of subcellular localization prediction showed that CsTPS1, CsTPS2, CsTPS3, CsTPS5, CsTPS6, CsTPS8, CsTPS9, CsTPS10,
CsTPPF were located in cytoplasm, CsTPS4, CsTPPC, $C S T P P D, C S T P P E$ in the nucleus, CSTPS7 in the plasma membrane, CsTPPA in chloroplast, and $C S T P P B$ in mitochondrion.

There were 11 TPS and 10 TPP genes in Arabidopsis and $11 T P S$ and 11 TPP genes in rice. A phylogenetic tree of TPS and TPP family members in cannabis, Arabidopsis and rice was constructed by the neighbor-joining (NJ) method of MEGA 7.0 software (Fig. 1). The figure shows that all genes are divided into three subgroups (Class I, Class II, and Class III). Class I includes cannabis CsTPS1-3, Arabidopsis AtTPS1-4, and rice OsTPS1. Class II includes cannabis CsTPS4-10, Arabidopsis AtTPS5-11, and rice OsTPS2-11. All Class III genes are TPP genes, including cannabis CSTPPA-F, Arabidopsis AtTPPA-J, and rice 


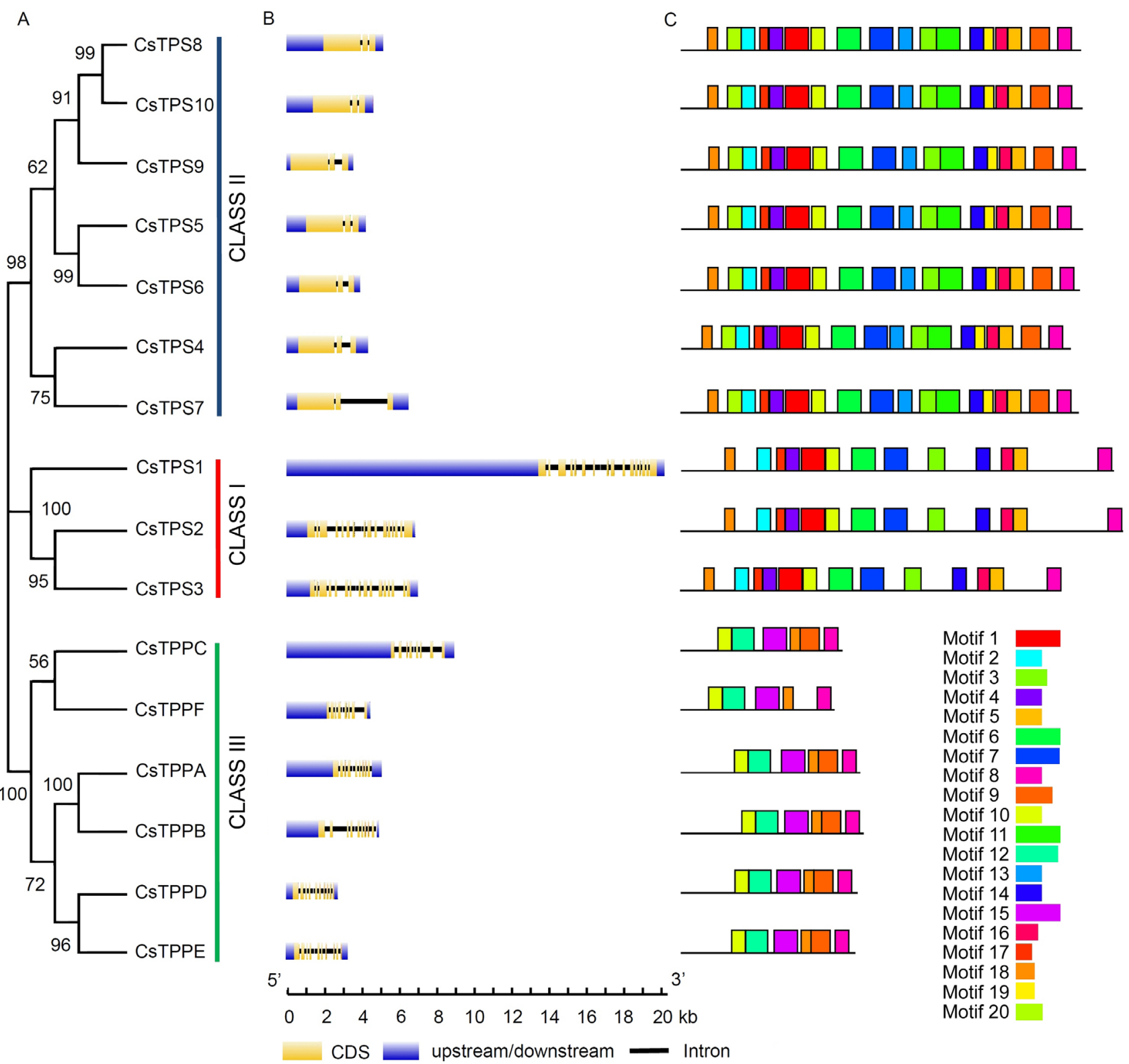

Fig. 2. Gene structure and motif distribution of the TPS and TPP families in cannabis. A - Phylogenetic tree of CsTPSs and CsTPPs; $B$ - the gene structure corresponding to CsTPSs and CsTPPs in the phylogenetic tree; $C$-Conservative motifs corresponding to CsTPSs and CsTPPs in the phylogenetic tree.

\section{OsTPP1-11.}

Compared with Arabidopsis, which is a dicotyledonous plant, the cannabis TPS gene is one less than the Arabidopsis $T P S$ gene, which occurs in the Class I subgroup, and there are also four fewer TPP genes in cannabis than in Arabidopsis.

Based on the gene structure analysis by GSDS 2.0 (Fig. 2), both TPS and TPP genes of cannabis contain exons and introns, but the number of exons in different CsTPSs and CsTPPS is very different. The exons of Class I, Class II, and Class III were 11,3 , and $8-10$, respectively, suggesting that the differences in gene structure between CsTPSS and CsTPPs may lead to their different functions, especially in Class I and Class II subfamilies. Although they are all CSTPS genes, there are huge differences in gene structure. Therefore, it is necessary to study the function of these genes further.

The motif types and permutations of TPS and TPP gene family members were analyzed by the online software MEME. As shown in Fig. 2, the number and species of the 20 predicted motifs were different between $T P S$ and TPP family members. Each member of the Class I subfamily contains 13 motifs, each member of the Class II subfamily contains 18 motifs, and each member of the Class III subfamily contains 6 motifs except CsTPPF. Only three motifs were found in all genes: motif 8 , motif 10 , and motif 18 .

TPS and TPP genes were distributed on all nine 


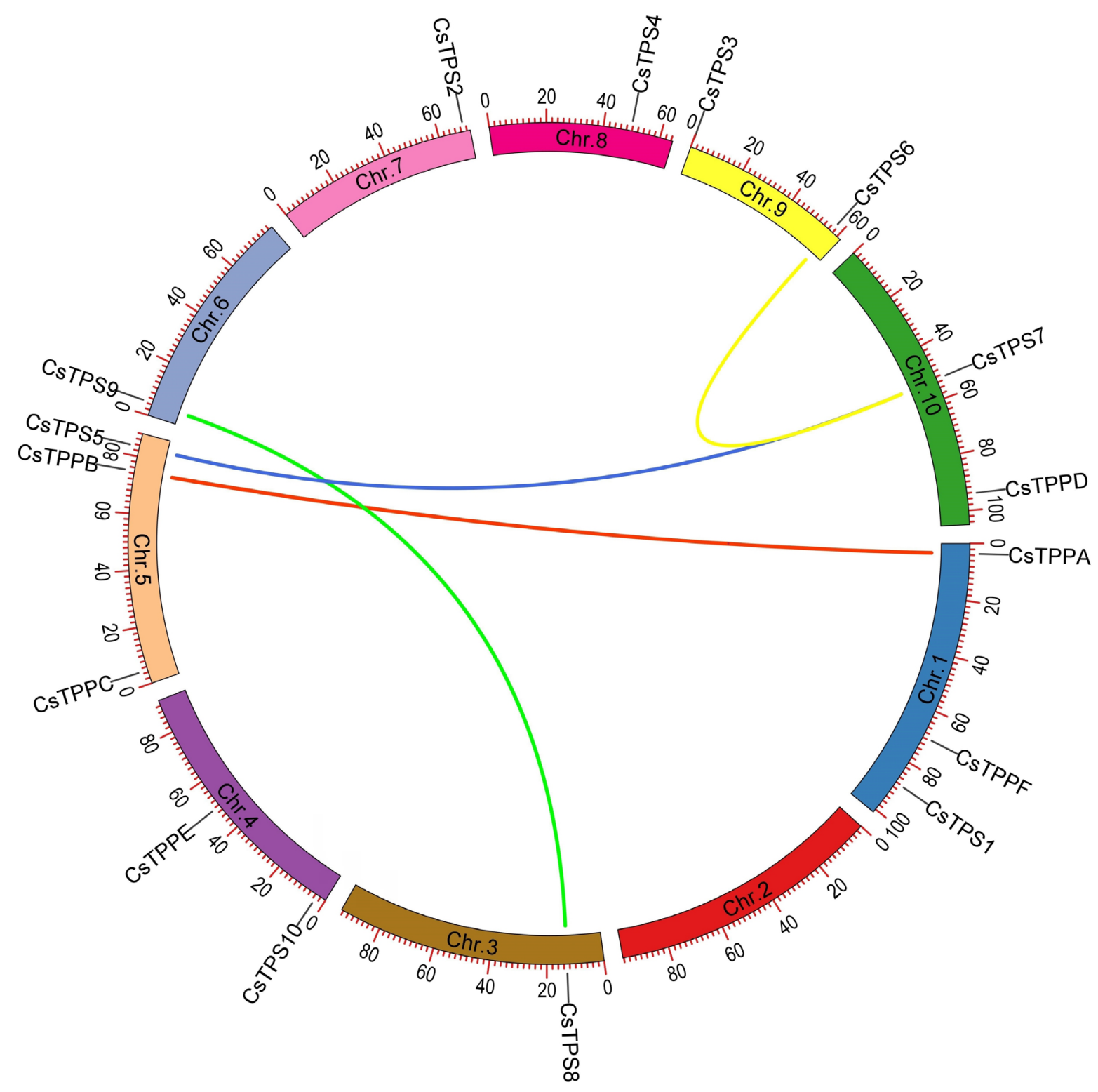

Fig. 3. Chromosomal location and gene replication relationship of TPS and TPP family genes in cannabis. The coloured lines in the circle represent the duplicated gene pairs.

chromosomes except chromosome 2 (Fig. 3). Among these genes, there were three on chromosomes 1 and 5, two on chromosomes 4, 9, and 10, and one on chromosomes 3 , 6, 7, and 8. CsTPSs and CsTPPS were randomly distributed on chromosomes, and there was no single distribution of CsTPSs or CsTPPS on one chromosome. For example, there are CsTPS1, CsTPPA, and CsTPPF on chromosome 1 and CsTPS5, CsTPPB, and CsTPPC on chromosome 5. The distribution of genes on chromosomes is not necessarily related to their position in the evolutionary tree.

Plant Genome Duplication Database (PGDD) was used for gene duplication analysis. The results showed that there were four pairs of genes that had gene duplication (Fig. 3, Table 2), and the duplication type was segmental duplication. Duplicated genes accounted for $43.75 \%$ of the total genes, indicating that gene duplication events played an important role in the amplification of TPS and $T P P$ families in cannabis, especially CsTPSs, which added three members through gene duplication. The number of TPP genes in cannabis is much less than the number of TPP genes in Arabidopsis, which may also be due to the loss caused by genome duplication.

In genetics, $\mathrm{Ka} / \mathrm{Ks}$ represents the ratio of the nonsynonymous substitution rate to the synonymous substitution rate of two protein-coding genes, which can determine whether there is selective pressure on genes. $\mathrm{Ka} / \mathrm{Ks}<1$ was considered purification selection, which indicates that natural selection eliminates harmful mutations and keeps the protein unchanged. $\mathrm{Ka} / \mathrm{Ks}>1$ is positive selection, which indicates that natural selection acts on the change of protein, makes the mutation site quickly fixed in the population and accelerates gene 
Table 2. Nonsynonymous substitution rate (Ka), synonymous substitution rate (Ks), $\mathrm{Ka} / \mathrm{Ks}$ analysis, and divergence times of duplicated CsTPSs and CsTPPS in cannabis.

\begin{tabular}{llllll}
\hline Duplicated pair & Duplicate type & Ka & Ks & Ka/Ks & Divergence time [Mya] \\
\hline CsTPS7/CsTPS6 & segmental duplication & 0.4429 & 0.5208 & 0.8504 & 17.36 \\
CsTPS7/CsTPS5 & segmental duplication & 0.3692 & 0.5904 & 0.6253 & 19.68 \\
CsTPS9/CsTPS8 & segmental duplication & 0.4035 & 0.4948 & 0.8155 & 16.49 \\
CsTPPB/CSTPPA & segmental duplication & 0.2624 & 0.6689 & 0.3923 & 22.30 \\
\hline
\end{tabular}

evolution. $\mathrm{Ka} / \mathrm{Ks}=1$ is neutral selection, which indicates that natural selection has no effect on mutation (Swanson et al. 2001, Hurst et al. 2002). To explore the selection mode of TPS and TPP genes in cannabis after duplication, $\mathrm{Ka}, \mathrm{Ks}$, and $\mathrm{Ka} / \mathrm{Ks}$ ratios were calculated by using the CDSs of duplicated gene pairs (Table 2). The $\mathrm{Ka} / \mathrm{Ks}$ ratios of the four pairs of duplicated genes in the TPS and TPP gene families were all less than 1 , which indicated that they carried out purification selection during duplication. The divergence time of duplicated gene pairs was calculated by the $\mathrm{T}=\mathrm{Ks} / 2 \mathrm{k}$ equation. The divergence time of the four pairs of genes was 16.49 - 22.30 million years ago (Mya), which was after the divergence of monocotyledons and dicotyledons (approximately $200 \mathrm{Mya}$ ) (Wolfe et al. 1989) and after the divergence of cannabis and $T$. orientale (approximately 52 Mya) (Gao et al. 2020).

Gene expression patterns can provide an important basis for research on gene function. Cannabis seedlings were treated with $\mathrm{NaCl}$, PEG-6000, and $4{ }^{\circ} \mathrm{C}$, and the expression patterns of the CsTPS and CsTPP gene families under salt, drought, and low-temperature stress were analyzed by RT-qPCR. The results showed that some CSTPS and CsTPP genes were induced to be expressed under abiotic stress, but there were differences among different genes (Fig. 1 Suppl.). CsTPS1, CsTPS6, CsTPS9, CsTPS10, CsTPPA, and CsTPPC were significantly upregulated under the three stresses, indicating that they may be important abiotic stresses regulatory genes. Among these genes, CsTPS1 was more upregulated under salt stress and low temperature, CsTPS9 was more upregulated under salinity and drought, and CSTPPA was more upregulated under salt stress than in other conditions, suggesting that the above three genes may play a key role in these stresses.

Some genes were significantly activated by one or two stresses; for example, CsTPS 2 was significantly upregulated under salt and drought stress, CsTPS 8 was significantly upregulated under salt stress, and CsTPS3, CsTPS5, and CSTPPB were significantly upregulated under low temperature. All differentially expressed genes were upregulated, and only CSTPS5 was downregulated by salt and drought stress, but there was no significant difference compared with $0 \mathrm{~h}$ at most time points. Although the expression of CSTPPF was upregulated at several time points under the three stresses, the expression of CSTPPF was also upregulated at 16 and $24 \mathrm{~h}$ in control, suggesting that $C S T P P F$ may be related to growth and development or time rhythm, and its relationship with abiotic stress needs to be studied further. The expression of CSTPS4, CsTPS7,
CsTPPD, and CsTPPE was not significantly induced, indicating that they had little relationship with abiotic stresses.

\section{Discussion}

In recent years, studying the classification, sequence characteristics, evolutionary characteristics, and functional prediction of plant gene families at the whole genome level has become an important means to fully understand gene families and carry out gene function research. The TPS and TPP families are gene families with important biological functions in plants. These genes are confirmed to be widely involved in the regulation of plant growth, development and metabolism, especially in improving stress resistance (Satoh-Nagasawa et al. 2006, Kosar et al. 2019).

In this study, 10 CsTPSs and 6 CsTPPs were identified from the genome of cannabis. CsTPSs contained complete Glyco transf 20 and Trehalose PPase domains, and $C S T P \bar{P} S$ contained only Trehalose PPase domains. The number of ten CsTPSs was less than the number of Arabidopsis (11) (Yang et al. 2012), rice (11) (Ge et al. 2008), and cotton (14) (Mu et al. 2016) and more than the number of loti (9) (Jin et al. 2016), Brachypodium distachyon (9) (Wang et al. 2019), and potato (8) (Xu et al. 2017). Six CsTPPs were less abundant than those of Arabidopsis (10) (Vandesteene et al. 2012), rice (11) (Ge et al. 2008), and Brachypodium distachyon (10) (Wang et al. 2019).

In this study, CsTPSs and CsTPPs were clustered into three subfamilies. CsTPSs were divided into Class I and Class II subfamilies, and all class III subfamilies were CsTPPs. The clustering result was consistent with the clustering result of other plants. In terms of gene structure, Class I and Class III contained more exons, indicating that they have more complex structural characteristics. However, class II, which is the same as CsTPSs, contains few exons, which is consistent with the gene structure of TPS and TPP in other plants. The gene structure and motif of Class I and Class II members are basically the same (Fig. 2), which is shown in the exact same number of exons and motif types of each class. There were slight differences in gene structure and motifs between Class III members. The TPS and TPP gene families are highly conserved in evolution and structure, which can provide some reference for the study of TPS and TPP gene function in cannabis.

In the evolutionary process, with the different 
duplication selection events experienced by different species, homologous genes have different degrees of differentiation. The common types are neofunctionalization, subfunctionalization, gene functional redundancy, and gene loss (Force et al. 1999). The number of TPS genes in cannabis was more or less similar than that in other plants. However, the number of TPP genes was 4, 5, and 4 less than the number of TPS genes of Arabidopsis, rice, and Brachypodium distachyon, respectively, which may be due to the loss of some TPP genes after gene duplication, because a newly duplicated gene can be either lost or fixed in the chromosome by genetic drift or natural selection (Lynch et al. 2001).

There were four gene duplications in CsTPS and CsTPP genes: CsTPS5 and CsTPS7, CsTPS6 and CsTPS7, CsTPS8 and CsTPS9, and CsTPPA and CsTPPB, suggesting that gene duplication events played an important role in the amplification of TPS and TPP families in cannabis. The results of expression analysis under abiotic stress showed that CsTPS7 was not induced to be expressed under the three stresses, while its duplicated genes CsTPS5 and CsTPS6 were expressed under all three stresses, indicating that gene duplication led to the divergence of some cannabis TPS genes into new functions.

$T P S$ and TPP are recognized as genes that can improve plant stress resistance (Delorge et al. 2014, Kosar et al. 2019). With the development of molecular biology, the stress-resistant functions of many TPS and TPP family members in Arabidopsis have been elucidated. Overexpression of AtTPS1 results in increased trehalose content, and transgenic plants show strong drought tolerance (Avonce et al. 2004). AtTPPD-deficient mutants are sensitive to salt stress, while AtTPPD overexpressing plants are more tolerant to salt stress (Krasensky et al. 2014). AtTPPF deletion mutation results in a drought-sensitive phenotype of Arabidopsis, while its overexpression lines show significant drought tolerance and trehalose accumulation (Lin et al. 2019). AtTPPI can enhance the drought resistance of Arabidopsis by regulating the stomatal opening and root structure (Lin et al. 2020). These results indicate that genes in the different subgroups of TPS and TPP have similar or different functions.

In this study, 11 cannabis TPS and TPP genes, CsTPS1, CsTPS2, CsTPS3, CsTPS5, CsTPS6, CsTPS8, CsTPS9, CsTPS10, CsTPPA, CsTPPB and CsTPPC, were significantly induced by at least one abiotic stress, accounting for $68.75 \%$ of the total gene family. CSTPS1 was significantly upregulated under three abiotic stresses, which is consistent with the obvious function of its Arabidopsis and rice homologous genes AtTPS1 and OsTPS1 (Ge et al. 2008) under abiotic stresses. CsTPPA and $O S T P P 1$ have similar positions in the evolutionary tree, and both exhibit certain abiotic stress regulation functions (Zhang et al. 2017), suggesting that the TPS1 and TPP1 genes have similar functions in different species.

In the phylogenetic tree, CsTPS1, CsTPS2, and CsTPS3 were in the same subfamily and are clustered together. The results of expression analysis showed that these three genes were upregulated to varying degrees under the three abiotic stresses, and the relative expression at most time points was significantly different from the relative expression at $0 \mathrm{~h}$, indicating that some genes with similar positions in the phylogenetic tree may have similar functions. The gene pairs CsTPS4 and CsTPS7, CsTPS8 and CsTPS10, CsTPPD and CSTPPE were very close in the phylogenetic tree. Similarly, the two genes of these gene pairs have similar functions. The gene pair CsTPS5 and CsTPS6 was clustered together in the phylogenetic tree. Although they were expressed under three abiotic stresses, their expression trend was opposite under salt stress and drought stress. The expression patterns of CSTPPA and $C S T P P B$ were the same only under cold stress, but they were significantly different under salt and drought stresses. These results indicate that the two groups of genes with similar positions in the phylogenetic tree have partial functional divergence, which can be used as references for each other in the study of gene function.

\section{Conclusions}

In this study, systematic analysis of TPS and TPP family genes have been performed in cannabis, and ten CsTPS and six CSTPP genes were identified at the genomic level. The TPS and TPP family members of cannabis, Arabidopsis, and rice were classified as three subgroups based on an evolutionary tree. There were four pairs of CsTPSs and CsTPPs that had gene duplication, indicating that gene duplication events played an important role in the amplification of TPS and TPP families in cannabis. The expression patterns under various abiotic stresses showed that most of the TPS and TPP genes may be involved in stress tolerance. In particular, CsTPS1, CsTPS9, and CsTPPA might be more induced by at least one abiotic stress, indicating that these three genes may play key roles in abiotic stresses. This study lays a foundation for further study on the biological functions of CsTPSs and CsTPPS.

\section{References}

Andre, C.M., Hausman, J.F., Guerriero. G.: Cannabis sativa: the plant of the thousand and one molecules. - Front. Plant. Sci. 7: 19, 2016.

Avonce, N., Leyman, B., Mascorro-Gallardo, J., Van Dijck, P., Thevelein, J.M., Iturriaga, G.: The Arabidopsis trehalose-6-P synthase AtTPS1 gene is a regulator of glucose, abscisic acid, and stress signaling. - Plant Physiol. 136: 3649-3659, 2004.

Avonce, N., Mendoza-Vargas, A., Morett, E., Iturriaga, G.: Insights on the evolution of trehalose biosynthesis. - BMC Evol. Biol. 6: 109, 2006.

Chen, C.J., Chen, H., Zhang, Y., Thomas, H.R., Frank, M.H., He, Y.H., Xia, R.: TBtools: an integrative toolkit developed for interactive analyses of big biological data. - Mol. Plants 13: 1194-202, 2020.

Delorge, I., Janiak, M., Carpentier, S., Dijck, P.V.: Fine tuning of trehalose biosynthesis and hydrolysis as novel tools for the generation of abiotic stress tolerant plants. - Front. Plant Sci. 5: 147, 2014.

Dong, Y.J., Zhang, Q., Dai, X.L., He, M.R.: Effects of potassium chloride and nitric oxide on growth and physiological 
characteristics of winter wheat under salt stress. - Biol. Plant. 64: 258-265, 2020

Du, L., Qi, S., Ma, J., Xing, L., Fan, S., Zhang, S., Li, Y., Shen, Y., Zhang, D., Han, M.: Identification of TPS family members in apple (Malus $\times$ domestica Borkh.) and the effect of sucrose sprays on TPS expression and floral induction. - Plant Physiol. Biochem. 120: 10-23, 2017.

Force, A., Lynch, M., Pickett, F.B., Amores, A., Yan, Y., Postlethwait, J.: Preservation of duplicate genes by complementary, degenerative mutations. - Genetics 151: 1531-1531, 1999.

Gao, S., Wang, B., Xie, S., Xu, X., Zhang, J., Pei, L., Yu, Y., Yang, W., Zhang, Y.: A high-quality reference genome of wild Cannabis sativa. - Hort. Res. 7: 73, 2020.

Ge, L.F., Chao, D.Y., Shi, M., Zhu, M.Z., Gao, J.P., Lin, H.X.: Overexpression of the trehalose-6-phosphate phosphatase gene OSTPP1 confers stress tolerance in rice and results in the activation of stress responsive genes. - Planta 228: 191201, 2008.

Hackel, C., Zinkevich, T., Belton, P., Achilles, A., Reichert, D., Krushelnitsky, A.: The trehalose coating effect on the internal protein dynamics. - Phys. Chem. Chem. Phys. 14: 2727-2734, 2012.

Han, B., Fu, Li., Zhang, D., He, X., Chen, Q., Peng, M., Zhang, J.: Interspecies and intraspecies analysis of trehalose contents and the biosynthesis pathway gene family reveals crucial roles of trehalose in osmotic-stress tolerance in cassava. - Int. J. mol. Sci. 17: 1077, 2016.

Hu, H., Liu, H., Du, G., Yang, F., Deng, G., Yang, Y., Liu, F.: Fiber and seed type of hemp (Cannabis sativa L.) responded differently to salt-alkali stress in seedling growth and physiological indices. - Indust. Crop. Prod. 129: 624-630, 2019.

Hu, X., Wu, Z.D., Luo, Z.Y., Burner, D.M., Pan, Y.B., Wu, C.W.: Genome-wide analysis of the trehalose-6-phosphate synthase (TPS) gene family and expression profiling of ScTPS genes in sugarcane. - Agronomy 10: 969, 2020.

Hurst, L.D.: The $\mathrm{Ka} / \mathrm{Ks}$ ratio: diagnosing the form of sequence evolution. - Trends Genet. 18: 486-487, 2002.

Jin, Q., Hu, X., Li, X., Wang, B., Wang, Y., Jiang, H., Mattson, N., Xu, Y.: Genome-wide identification and evolution analysis of trehalose-6-phosphate synthase gene family in Nelumbo nucifera. - Front. Plant Sci. 7: 1445, 2016.

Kosar, F., Akram, N.A., Sadiq, M., Al-Qurainy, F., Ashraf, M.: Trehalose: a key organic osmolyte effectively involved in plant abiotic stress tolerance. - J. Plant Growth Regul. 38: 606-618, 2019.

Krasensky, J., Broyart, C., Rabanal, F.A., Jonak, C.: The redoxsensitive chloroplast trehalose-6-phosphate phosphatase AtTPPD regulates salt stress tolerance. - Antioxid. Redox Sign. 21: 1289-1304, 2014.

Lerbret, A., Bordat, P., Affouard, F., Guinet, Y., Hedoux, A., Paccou, L., Prevost, D., Descamps, M.: Influence of homologous disaccharides on the hydrogen-bond network of water: complementary Raman scattering experiments and molecular dynamics simulations. - Carbohydrate Res. 340: $881-887,2005$.

Librado, P., Rozas, J.: DnaSP v5: a software for comprehensive analysis of DNA polymorphism data. - Bioinformatics 25: 1451-1452, 2009.

Lin, M., Jia, R., Li. J., Zhang, M., Chen, H., Zhang, D., Zhang, J., Chen, X.: Evolution and expression patterns of the trehalose6-phosphate synthase gene family in drumstick tree (Moringa oleifera Lam.). - Planta 248: 999-1015, 2018.

Lin, Q., Wang, S., Dao, Y., Wang, J., Wang, K.: Arabidopsis thaliana trehalose-6-phosphate phosphatase gene TPPI enhances drought tolerance by regulating stomatal apertures. - J. exp. Bot. 71: 4285-4297, 2020.

Lin, Q., Yang, J., Wang, Q., Zhu, H., Chen, Z., Dao, Y., Wang, K.: Overexpression of the trehalose-6-phosphate phosphatase family gene $A t T P P F$ improves the drought tolerance of Arabidopsis thaliana. - BMC Plant Biol. 19: 381, 2019.

Livak, K.J., Schmittgen, T.D.: Analysis of relative gene expression data using real-time quantitative PCR and the

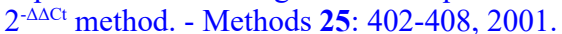

Lynch, M., O'Hely, M., Walsh, B., Force, A.: The probability of preservation of a newly arisen gene duplicate. - Genetics 159:1789-1804, 2001.

Mahajan, S., Tuteja, N.: Cold, salinity and drought stresses: an overview. - Arch. Biochem. Biophys. 444: 139-158, 2005.

Morabito, C., Secchi, F., Schubert, A.: Grapevine TPS (trehalose6-phosphate synthase) family genes are differentially regulated during development, upon sugar treatment and drought stress. - Plant Physiol. Biochem. 164: 54-62, 2021.

Mu, M., Lu, X.K., Wang, J.J., Wang, D.L., Yin, Z.J., Wang, S., Fan, W.L., Ye, W.W.: Genome-wide identification and analysis of the stress-resistance function of the TPS (Trehalose-6Phosphate Synthase) gene family in cotton. - BMC Genet. 17: 54, 2016.

Paul, M.J., Primavesi, L.F., Jhurreea, D., Zhang, Y.: Trehalose metabolism and signaling. - Annu. Rev. Plant Biol. 59: 417441, 2008

Pretzsch, C.M., Freyberg, J., Voinescu, B., Lythgoe, D., Horder, J., Mendez, M.A., Wichers, R., Ajram, L., Ivin, G., Heasman, M., Edden, R.A.E., Williams, T., Murphy, D.G.M., Daly, E., McAlonan, G.M.: Effects of cannabidiol on brain excitation and inhibition systems; a randomised placebo-controlled single dose trial during magnetic resonance spectroscopy in adults with and without autism spectrum disorder. Neuropsychopharmacology 44: 1398-1405, 2019.

Satoh-Nagasawa, N., Nagasawa, N., Malcomber, S., Sakai, H., Jackson, D.: A trehalose metabolic enzyme controls inflorescence architecture in maize. - Nature 441: 227-230, 2006.

Skoglund, G., Nockert, M., Holst, B.: Viking and early middle ages northern Scandinavian textiles proven to be made with hemp. - Sci. Rep. 3: 2686, 2013.

Sundaramurthi, P., Patapoff, T.W., Suryanarayanan, R.: Crystallization of trehalose in frozen solutions and its phase behavior during drying. - Pharm. Res. 27: 2374-2383, 2010.

Swanson, W.J., Yang, Z., Wolfner, M.F., Aquadro, C.F.: Positive Darwinian selection drives the evolution of several female reproductive proteins in mammals. - Proc. nat. Acad. Sci. USA 98: 2509-2514, 2001.

Vandesteene, L., Lopez-Galvis, L., Vanneste, K., Feil, R., Maere, S., Lammens, W., Rolland, F., Lunn, J.E., Avonce, N., Beeckman, T., Van Dijck, P.: Expansive evolution of the trehalose-6-phosphate phosphatase gene family in Arabidopsis. - Plant Physiol. 160: 884, 2012.

Vij, S., Tyagi, A.K.: Emerging trends in the functional genomics of the abiotic stress response in crop plants. - Plant Biotechnol. J. 5: 361-380, 2010.

Wang, S., Ouyang, K., Wang, K.: Genome-wide identification, evolution, and expression analysis of TPS and TPP gene families in Brachypodium distachyon. - Plants 8: 362, 2019.

Wolfe, K.H., Gouy, M., Yang, Y.W., Sharp, P.M., Li, W.H.: Date of the monocot-dicot divergence estimated from chloroplast DNA sequence data. - Proc. nat. Acad. Sci. USA 86: 62016205, 1989.

Xie, D.W., Wang, X.N., Fu, L.S., Sun, J., Zheng, W., Li, Z.F.: Identification of the trehalose-6-phosphate synthase gene family in winter wheat and expression analysis under 
conditions of freezing stress. - J. Genet. 94: 55-65, 2015.

Xu, Y., Wang, Y., Mattson, N., Yang, L., Jin, Q.: Genomewide analysis of the Solanum tuberosum (potato) trehalose6-phosphate synthase (TPS) gene family: evolution and differential expression during development and stress. - BMC Genomics 18: 926, 2017.

Yang, H.L., Liu, Y.J., Wang, C.L., Zeng, Q.Y.: Molecular evolution of trehalose-6-phosphate synthase (TPS) gene family in Populus, Arabidopsis and rice. - PLoS ONE 7: e42438, 2012.

Yang, Y., Ma, K., Zhang, T., Li, L., Wang, J., Cheng, T., Zhang,
Q.: Characteristics and expression analyses of trehalose-6phosphate synthase family in Prunus mume reveal genes involved in trehalose biosynthesis and drought response. Biomolecules 10: 1358, 2020.

Zhang, Q., Chen, Q., Wang, S., Hong, Y., Wang, Z.: Rice and cold stress: methods for its evaluation and summary of cold tolerance-related quantitative trait loci. - Rice 7: 24, 2014.

Zhang, Z., Li, J., Li, F., Liu, H., Yang, W., Chong, K., Xu, Y.: OsMAPK3 phosphorylates OsbHLH002/OsICE1 and inhibits its ubiquitination to activate OSTPPI and enhances rice chilling tolerance. - Dev. Cell 43: 731-743, 2017. 